To be presented at the 31st Annual Convention of the American Institute of Electrical Engineers, Detroit, Mich., June 24, 1914, under the auspices of the Electrophysics Committee.

Copyright 1914. By A.I.E.E.

(Subject to final revision for the Transactions.)

\title{
SPHERE GAP DISCHARGE VOLTAGES AT HIGH FREQUENCIES
}

\author{
BY J. CAMERON CLARK AND HARRIS J. RYAN
}

Abstract of Paper

The paper describes a series of experiments made by the authors to determine the values of steady high-frequency, high-voltage currents required to discharge between seveninch copper spheres in air, at ordinary temperatures and barometric pressures. A $15-\mathrm{kw}$. arc generator was used as the source of high-frequency sustained high voltage, and the apparatus employed in securing and measuring currents of 123,000, 255,000 and 612,500 cycles is described in detail.

The sphere-gap standard consisted of electrolytic copper spheres mounted on the ends of brass tubes in treated wooden frames, and the gap lengths were accurately determined by means of calipers and micrometer screw or steel scale.

The results obtained are given in Tables II and III and are also charted in curves I and II, Fig. 5. The 123,000-cycle values show a right line relation between sphere gaps and discharge voltage, which line, when extended, passes through the origin. The high-frequency voltages are almost uniformly $4.5 \mathrm{kv}$. below the 25 -cycle voltages observed by Chubb and Fortescue.

\section{INTRODUCTION}

$\mathrm{T}$

HE predatory character of high-frequency currents due to accidental causes on transmission lines is now generally recognized, especially with reference to insulation. The modern high-power radiotelegraph has encountered occasional diffculty in the maintenance of insulation that is also stressed with high-frequency high voltage. These facts have emphasized the need of more quantitative knowledge of high-frequency phenomena. To obtain this knowledge many laboratory studies and measurements will have to be made to provide more conclusive data and trained mental equipments required to interpret causes of insulation failure and to eliminate them.

To aid in these matters we have undertaken to determine the values of steady high-frequency high voltages required to discharge between seven-inch (17.7-cm.) copper spheres, in air, at ordinary temperatures and barometric pressures. 
High-Frequency Sustained High-Voltage Source

A 15-kw. Poulsen-Federal arc generator was used. See Fig. 1. The arc is drawn in a powerful magnetic field and surrounded by water-cooled illuminating gas. A slowly-rotating carbon rod forms the cathode, and a water-cooled bronze lug forms the anode. The characteristics of this particular arc have not as yet been fully determined. At 17.6 amperes direct current through the arc and 12.5 amperes alternating current at 123,000 cycles per second in the oscillating circuit the arc is stable at 1200 continuous volts. Under these conditions the resistance ballast at $R$ is all cut out, and the power

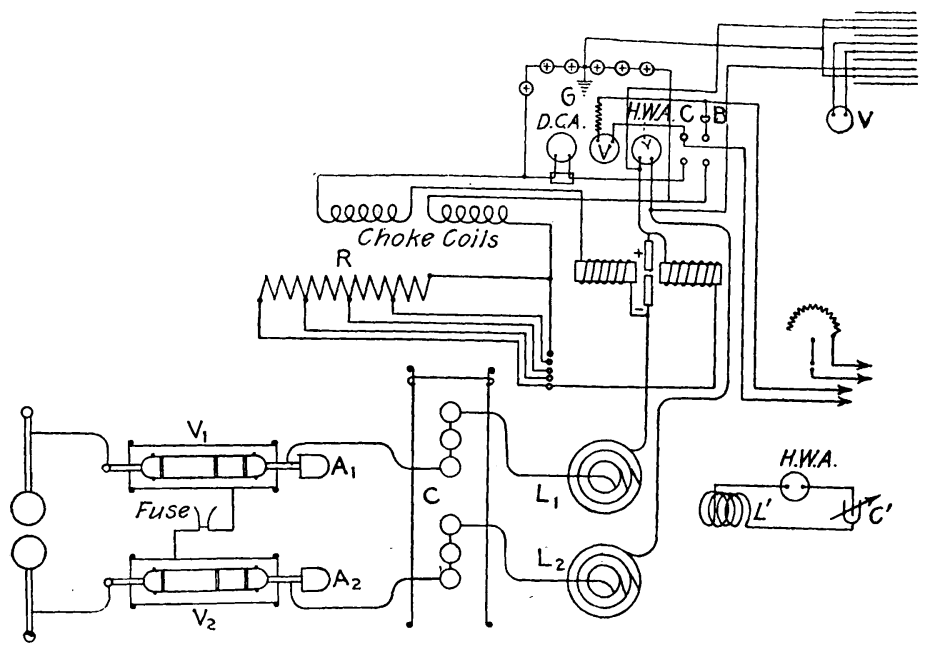

FIG. 1

consumed in the oscillating circuit is chiefly that lost in the carborundum safety resistance rods, $S R$, in series with the sphere gap. This power amounts to about one $\mathrm{kw}$. There are two of these rods, each $8 \frac{3}{4}$ in. $\left(222.25 \mathrm{~mm}\right.$.) long and $\frac{3}{4}$ in. $(19.05$ $\mathrm{mm}$.) in diameter. The charging current taken by the seveninch $(17.7-\mathrm{cm}$.) spheres at 123,000 cycles is sufficient to maintain the rods at a bright cherry-red temperature.

The insulation of the field coils of the arc generator must be extra heavy as the duty put upon it is severe. The outer terminals of the coils are connected to the anode and cathode of the arc. Correspondingly, the inner terminals are connected to the inductances that protect the direct-current generator 
from "static". Adjustable static arrester gaps shunt these field coils to short circuit the "kick" of the field when the arc breaks. The arc will break occasionally, stopping the current through the field coils with suddenness. The discharge from these field coils will puncture their own insulation and that of the direct-current generators if such inductances and dischargers are not used. For greater safety in this regard, a series of carbonfilament incandescent lamps connects across the terminals of the generator. The middle of the series is grounded. Two 7.5-kw., 600-volt direct-current generators furnish current to the arc-generator. They are connected in series, with their mid-connection grounded. The first machine is separately excited from a 125 -volt source. The 600 -volt field of the second generator is excited with current taken from the armature of the first generator. Thus a rheostat in the field of the first generator controls the total direct-current supply voltage. This rheostat is mounted so that one can conveniently use it to adjust the direct-current supply voltage when he is starting the arc generator or adjusting its action.

The arc generator equipment includes one 50-ampere, directcurrent ammeter showing the average direct current drawn from the continuous-current supply generators; one 30-ampere Hartmann and Braun " hot-band " ammeter measuring the highfrequency amperes circulating in the oscillating circuit; one voltmeter for reading the continuous-current supply voltage; and a high-frequency voltage gage connected across the arc to enable one to know the voltage duty demanded of the arc to set up a given current in the oscillating circuit. The last instrument is casily made by connecting a one-ampere hotwire ammeter in series with two pairs of common tin sheets $\frac{1}{2}$ in. (12.7 mmm.) scparation, functioning as a condenser, and connected across the arc. The charging current taken by such condenser as indicated by the hot-wire ammeter is roughly proportional to the high-frequency voltage duty demanded of the arc. Such voltage is decidedly of irregular wave form so that this instrument functions simply as a voltage gage.

The inductance in the oscillating circuit is made up of approximately $2000 \mathrm{ft}$. $\left(609.6 \mathrm{~m}\right.$.) of $\frac{1}{4}$-in. $(6.3-\mathrm{mm}$.) soft copper tubing, forming six helices of 25 turns each. Each helix is mounted on six hard-rubber rods. The helices have graded diameters so that they may be telescoped to form one six-helix inductance or a pair of inductances of three helices each. The 
travel of the turns is the same for all helices,-an important matter when these large inductances are to be formed by telescoping. When the travels are not the same, i.e., when they reverse from layer to layer as in the ordinary solenoid, the distributed capacity of the inductance is broken into chunks causing turbulent interference with the growth of. the oscillating current. In a nest of helices the circuit leads through the first from the bottom turn to the top turn, thence downward to the bottom turn of the next helix, then out at the top, and again in at the bottom of the next, and so on through the whole set. Oscillating currents are easily driven through inductances of this sort when connected in series with suitable condensers. Even with this favorable construction the helices may not be nested in a single set because the duty demanded of the arc will easily exceed its voltage capacity bcfore a fair value of high-frequency current has been set up. It is necessary to mount the helices in two sets, preferably of equal inductances. Then, when a single condenser is connected symmetrically between the two inductances, and in series therewith, the phase displacements that the oscillating currents encounter with respect to the arc are opposed and balanced. The high-frequency voltage duty demanded of the are is correspondingly light. Without resorting to delicate balancing, 50,000 volts and 12.5 amperes at a frequency of 123,000 cycles per second may be developed in this apparatus. With greater care in balancing than we have so far employed, higher oscillating voltages can doubtless be developed.

The main condenser in the oscillating circuit is made up as shown at $C$ in the diagram of the complete outfit given in Fig. 1. Each electrode is formed of cylinders of galvanized sheet iron seven ft. (2.1 m.) long and five in. $(127 \mathrm{~mm}$.) in diameter, equipped with hemispherical ends. They are also equipped with hooks and eyes so that chains of parallel cylinders may thus be formed. The capacity can be increased or decreased by attaching and detaching one or more of these cylinders from each of the two electrode chains. This feature enables the capacity in the oscillating circuit and, therefore, the frequency, to be varied over wide limits. At present this main condenser is equipped with a chain of four cylinders at each electrode. The chains hang in a vertical plane between a pair of neutral plates forming a hood about the capacity cylinders as shown in Fig. 1. The frames of these plates are made of common 
PLATE XLV

A. I. E. E.

VOL. XXXIII, NO. 6

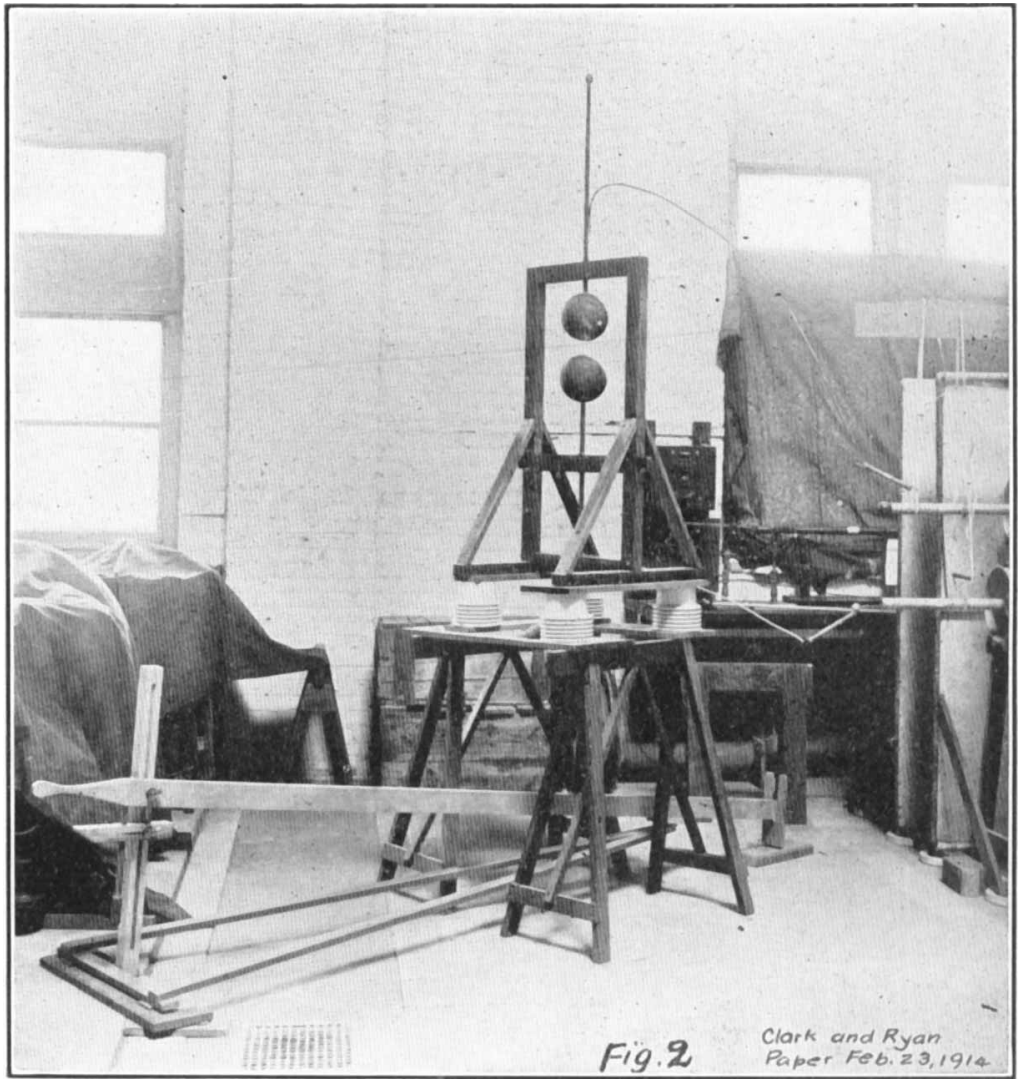

FIG. 2 





FIG. 3



FIG. 4 

galvanized iron pipe, $\frac{3}{4}$ in. (19.05 mm.) internal diameter; the bodies of the plates are constructed of galvanized iron, 23-mil sheets. The plates are tied together with a conductor. They are insulated from ground for a small portion of the total oscillating voltage, for unless special care is taken there is some unbalancing that sets up a potential between the plates and ground. The illustrations, Figs. 2, 3 and 4, give an idea of the actual manner in which the above parts of the generating equipment are formed and arranged. To the capacity of the main condenser there is necessarily added the capacity of the concentric-cylinder voltmeter condenser, $V_{1} V_{2}$, to be considered later.

In the final stage of the present undertaking, the number of helices was doubled by placing the six copper tubular helices in one nest and by the addition of a set of six helices of the same size, in which the conductor used is a "flat magnet wire" 0.34 by 0.04 , in $(8.6$ by $1 \mathrm{~mm}$.) in section. The latter set was made up, primarily, to serve as a protective inductance at the main terminal of a low-frequency high-voltage series of transformers, the other main terminal being grounded. However, by giving the helices of this set proper dimensions they could be used along with the regular set for the generation and measurement of higher voltages at lower frequencies in the standardization of the sphere-gap voltmeter. When using the largest capacity and self-induction that the equipment thus provided, 12.5 amperes at 100,000 volts and 85,000 cycles per second are produced in the oscillating circuit. Correspondingly when the capacity is reduced to the lowest practicable limit, viz., that of the voltmeter condenser and the self-induction is limited to that of the pair of small innermost helices, 8.1 amperes and 13,000 volts at 612,500 cycles are produced.

The carborundum protective resistance in series with the sphere-gap voltmeter could not be used at frequencies much above 123,000 cycles. This is due to the magnitude of the charging currents inevitably taken by the spheres and their connections, which cause excessive losses in resistances proportioned so as to limit properly the 1200 -volt direct power-current that follows a discharge across the gap. At higher frequencies a fine-wire fuse, guarded to prevent corona, was used to limit the injury produced by the power currents. The capacity of the fuse must necessarily be large enough to carry the charging currents taken by the spheres and their connections. On 
this account such fuse capacity cannot be made small enough entirely to prevent injury to the spheres. To overcome this difficulty in the later stages of the work, when frequencies higher than 123,000 cycles were used, the Fortescue-Chubb-Farnsworth mounting for the spheres had to be slightly modified. The brass rods supporting the seven-in. $(17.7-\mathrm{cm}$.) spheres were terminated in shallow cups with inside surfaces fitting them. The spheres were then mounted upon these cups, being held in place by thin sheet brass halters and rubber bands. These changes in the sphere gap standard do not appear to be of material consequence as affecting its integrity for the present class of measurements, while they permit the spheres to be rotated when their adjacent surfaces have been injured so as to bring into use new and uninjured surfaces. By this means the reliable useful life of the spheres is prolonged to such an extent that their renewal cost becomes nominal.

\section{High-Frequency Meter}

In the present undertaking a reliable frequency or wave meter is of prime importance. The frequency meter employed was made by connecting an inductance standard in series with a variable condenser of reliable make and of a type commonly employed in radio work. Three entirely independent methods were employed for standardizing this meter.

1. By Calculation: In this method the value of the inductance was calculated. The conductor used in its construction was enamel-insulated litzen draht, silk jacketed. When constructed, the dimensions were obtained with some care. Perry's formula ${ }^{1}$ and Doggett's method ${ }^{2}$ were used to calculate independent values of the inductance. The results obtained were 879.0 and 882.5 microhenrys. The variable condenser was compared with a standardized condenser of the Federal Telegraph Company. It was also compared with an air-core. standard formed of two concentric brass tubes. The scale of the condenser was found to be true and linear. Its total capacity at the 180-deg. setting compared with the Federal standard was found to be 0.00405 microfarad and with the brass tube standard 0.00407 microfarad.

1. Perry's Approximate Self-Inductance Formula. Bulletin, Bureau of Standards. Vol. 8, No. 1, p. 136. Jan. 1, 1912.

2. Leonard A. Doggett. The Inductance of Air-Cored Solenoids. Electrical World, Vol. 63, p. 259, January 31, 1914. 
II. By Direct Measurement, as follows: At a definite setting of the wave meter the current through and the voltage across the terminals of the meter condenser were read. A one-ampere Hartmann and Braun hot-band ammeter was used for the current and a Kelvin multicellular electrostatic voltmetcr for the voltage. The latter instrument is essentially an air-core condenser. Its capacity could be determined conveniently and accurately by noting the change in the frequency meter reading that was caused by its presence. An exact correction on its account could therefore be made. The frequency was then calculated from the simple and well known relation of voltage current and capacity. This value was found to be 123,000 cycles to check with 122,500 cycles obtained by method I.

III. The wave meter was then taken to the factory of the Federal Telegraph Company in Palo Alto. There, through the courtesy of Mr. Ralph Beal, it was compared with the wave meter that had been standardized by the Burcau of Standards. The two wave meters were found to be in close agreement, differing only by $\frac{1}{4}$ per cent.

For higher frequencies the wave meter was recalibrated by method II to eliminate errors that appear duc to the internal capacity of the wave meter inductance.

\section{High-Frequency High-Voltage Meters}

The high voltages generated in the main condenser, $C$, Fig. 1, of the oscillating circuit, contain pure sine waves of a single frequency only. The rough pulsating voltages of the generator arc are completely absorbed by the main inductances, $L_{1} L_{2}$, in the oscillating circuit. A reliable voltage meter was, therefore, made out of a suitable condenser of known capacity connected in series with a hot-band ammeter that is free of frequency effects.

For balancing purposes and to employ convenient dimensions in other respects, it was found best in the present work to make up and use two of these condenser-type voltage meters. The manner of their construction is shown at $V_{1} V_{2}$ in Fig. 1. They were made in principle the same as that used by Chubb and Fortescue in standardizing the Farnsworth-Fortescue sphere gap voltmeter. ${ }^{3}$ Each condenser was made concentric and near to these specified dimensions: Outer cylinder: inner diameter 11.0 in. $(27.9 \mathrm{~cm}$.) length 72 in. $(1.8 \mathrm{~m}$.). Inner cylinder: outer diameter 7.0 in. $(17.7 \mathrm{~cm}$.), gross length, not including wood-

3. L. W. Chubb and C. Fortescue, Proc. A.I.E.E., Feb. 1913, p. 627. 
mounted hemispherical tinfoil-covered end caps, $61.0 \mathrm{in.}(1.54 \mathrm{~m}$.) Two central sections effective for use; one, length $12 \mathrm{in}$. $(30.4 \mathrm{~cm})$. the other 24 in. $(60.9 \mathrm{~cm}$.) ; total when connected in multiple 36 in. $(91.4 \mathrm{~cm}$.). These cylinders were constructed of 23 -mil galvanized iron. The inner cylinders were carried on a nine ft. $(2.7-\mathrm{m}$.$) length of common 13 / 4-\mathrm{in} .(3.44-\mathrm{cm}$.$) galvanized iron$ pipe. The outer cylinders were mounted one over the other, in an A-frame of wood, insulated on porcelain cleats from the cement floor against the small differential voltage; the inner cylinders were supported in position by sash cords. This support was made adjustable for centering by wedges. Errors due to eccentricity were measured and found to be negligible by noting changes in charging currents indicated by a synchronous commutator and portable direct-current galvanometer. The high-frequency, high voltages were applied to the inner cylinders by connecting to the pipe ends remote from the arc-generator. On the pipe ends facing the main inductances and the arc-generator, metal guard hoods were attached, $A_{1} A_{2}$. In them, fiveampere or one-ampere, hot-band ammeters were mounted and connected in series with the pipe and the central insulated condenser sections, so as to measure the charging current taken by such sections. The fronts of the hoods were made hemispherical of fine wire mesh to admit light. Through these, openings were cut to facilitate reading the ammeters from a distance by means of telescopes. An observer going near enough to read these instruments with the unaided eye would greatly disturb the balance of the oscillating system, cutting down the high frequency voltage sometimes by as much as 50 per cent, through the increased duty demanded of the arc when circulating current in an unbalanced circuit. Since the capacity values of the used sections of these condensers are made definite in terms of their linear dimensions by means of the guard ends, calculation was relied upon entirely to determine such values. In the upper cylinder the total capacity of the $36 \mathrm{in}$. $(91.4 \mathrm{~cm}$.) of used section was found to be 0.0001140 microfarads, and correspondingly, for the lower cylinder 0.0001145 microfarads. In obtaining the results herein reported, a five-ampere ammeter was used in series with the 0.0001145 microfarads in the lower cylinder and a oneampere ammeter in series with one third of the aggregate 0.0001140 microfarads available in the upper cylinder.

For safety, the outer upper and lower cylinders, which operate nearly at ground potential, are connected to the electrodes of a 
horn-gap bridged with a long fine-wire fuse. Occasionally when the arc was about to break an unbalancing surge would produce an extra high voltage that would discharge across the double two-in. (50.8 mm.) gaps between the voltmeter cylinders. If such discharge should strike the section of the inner cylinder that is connected through an ammeter it might be burned out before the fuse would interrupt the resulting short circuit. To prevent this, aluminum rings made from $1 / 4$-in. (6.3- $\mathrm{mm}$.) rod were fitted over the extreme ends of the inner cylinders. The discharges then took place from these rings, removing the danger from injury to the hot-band ammeters by short circuits. See Fig. 1. To lessen the spread of the electrical field set up from the hoods covering the ammeters, a sheet metal screen was mounted near them. This screen, the outer cylinders and the large hood over the cylinders, forming the main oscillating-circuit condenser, were all connected and operated near zero potential.

Stability in the production of high voltages by resonance from an arc-generator as a source, depends upon the constants of the oscillating circuit. These must be so established and related that oscillations can be set up at a single frequency only. In the equipment herein specified this condition could not be fully realized without encountering too much cost. The use of a single main condenser in series with two magnetically coupled inductances in the oscillating circuit fixed the frequency and gave ample stability. When the two concentric voltmeter condensers were added to the main condenser, it was found that the voltage duty put upon the arc-generator was somewhat increased. The oscillating freedom of the circuit was correspondingly impaired. This was no cause of difficulty in measuring voltages u1) to 50,000. Above 50,000 volts one or the other of the concentric voltmeter condensers was apt to flash over due to a tendency of the complex circuit to start local damped oscillations at much higher frequencies. The condenser voltmeter could not be used, therefore, to measure voltages much beyond 50,000.

\section{The Sphere-Gap Standard}

Electrolytic copper spheres were used and mounted on the ends of brass tubes in treated wood frames with proportional dimensions exactly as specified by Farnsworth and Fortescue. ${ }^{4}$ Satisfactory spheres may be obtained at small cost by selecting

4. S. W. Farnsworth \& C. L. Fortescue, Proc. A.I.E.E., Feb. 1913, p. 299. 
them for regularity of surface curvature from the commercial float ball product. Well-impregnated paraffined oak was used for the cross pieces through which the brass tubes pass to support the spheres. The rest of the frame was made of Oregon pine. Under the stress of high-frequency high voltage all dielectrics accept large charging currents. Ample and close electrode contact entirely free of all corona, must be made to lead these charging currents into the stressed dielectric so as to prevent burning or other injury. Accordingly the holes through the oak cross pieces were bushed with brass sleeves cemented to the wood by paraffin treatment. Without this precaution the frame will promptly be set afire. For balancing purpose we had to set this sphere gap standard rather high off the floor. For reasons given above it could not be operated with one terminal grounded as recommended by Fortescue, Chubb and Farnsworth. We also used a long wooden lever and screw jack to facilitate accurate gap settings. Gap lengths were determined by means of calipers and micrometer screw or steel scale.

Finally reference is made to the three illustrations Figs. 2, 3 and 4 , which give to the eye much additional information relating to the construction and arrangement of the equipment as employed and above described.

\section{Features of Unsteadiness in the High-Frequency High Voltages Generated in the Appara- tus as Used and Described Above}

The character of the high-frequency voltages was examined with the aid of the cathode ray cyclograph. The ray was given an alternating deflection in one plane magnetically, and in a quadrature plane, electrostatically. The magnet deflections were produced by the oscillating currents. Limitations in the behavior of the cathode ray tube made it necessary to use an air-core transformer in series with the oscillating circuit. The secondary of this transformer was short circuited through the current coils of the cyclograph and grounded. Thus the cathode ray was not subjected to the potential disturbances that occur when the deflecting coils are directly connected in series with the oscillating circuit adjacent to an ungrounded electrode of the generator-arc.

The ray was deflected electrostatically by "quadrants" charged with the potentials of spherical electrodes that were 
dipped into the powerful electrostatic field of the main condenser. The two alternating motions thus imparted to the ray being in quadrature, it was made to trace an ellipse on the observing screcn of the tube. One could then note the characteristic manner in which the magnitude of the high frequency voltage varied. The lowest current that could ordinarily be maintained in the oscillating circuit at 123,000 cycles was 3.0 and the highest 12.5 amperes, corresponding to 12.0 and 50.0 kilovolts respectively. The cyclograph indicated unsteady voltage fluctuations amounting to a maximum of 15 per cent, approximately, at 3.0 oscillating amperes. As the oscillating amperes increased the instantaneous voltage became quite steady, fluctuating less than 5 per cent, maximum. As the current approached 6.0 amperes producing 24.0 kilovolts, the voltage became quite unsteady. It was always difficult to operate the arc-generator at 6.5 amperes. At such current the hot-band ammeter reading fluctuates and the cyclograph indicates violent fluctuations in the oscillating current and voltage. Persistence of vision frequently caused two concentric cyclograms to appear upon the screen, one corresponding to a current of about six, and the other, seven amperes. Because of this characteristic of the arc no attempt at any time was made to determine sphere-gap discharge voltages at or near 6.5 amperes corresponding to 26.0 kilovolts. At seven amperes and upward to 12.5 amperes, the cyclograph again indicated steady instantaneous oscillating voltage, whenever the hot-band ammeter in the oscillating circuit produces steady readings.

In making sphere-gap voltage discharge observations the arcgenerator was operated and controlled so as to establish a definite high-frequency current in the oscillating circuit. While the sphere gap was shortened slowly to produce the discharge, the ammeters in the main oscillating and voltmeter condenser circuits were read. A set of observations was considered good only when the ammeters gave steady indications. In general, however, under the most steady operating conditions there are some slight and rather rapid fluctuations in the values of the current and, therefore, voltage. The ammeters can only indicate the effective average of such currents and voltages. On the other hand, it is the effective maximums of the slightly fluctuating voltages that actually produce most of the discharges between the spheres. In this manner variable errors are produced in the results, and some strategy is necessary to accomplish 
their approximate elimination. These errors always operate to produce apparent voltage values that are smaller than their corresponding true values. For obvious reasons the sphere gap can be shortened only at a slow rate. If it could be shortened at a very high rate and the process stopped at the exact length through which the discharge occurred, all settings would have ncarly cqual weight in determining the final true result. Under the actual working conditions in this equij)ment, it is hardly likely that the observed voltages are ever above the true vollages that actually produce the discharge between the spheres. Oc-

TABLE I

TyPical OBSERVATIONS

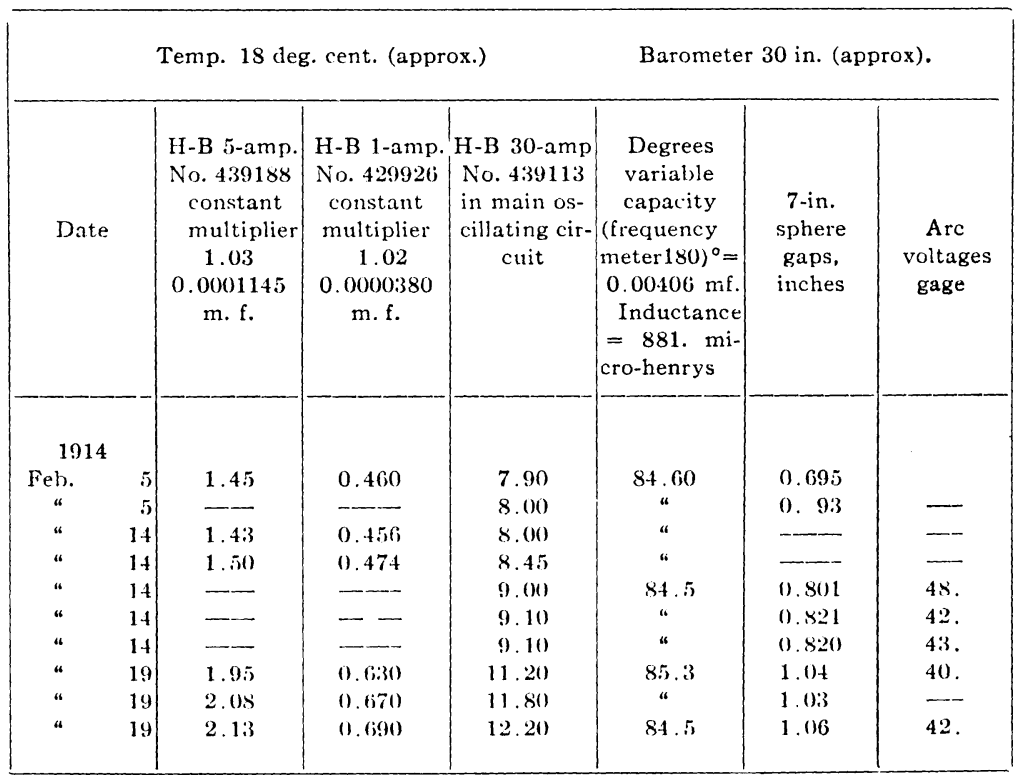

casionally, however, the readings of the instruments must. correspond much more mearly to the voltages applied to the spheres at the instant they discharge. The corresponding results will be least in error and must have most weight in determining the final and true relation between voltages and spark gap lengths.

\section{Discussion of REsults}

Some of the original observations are given in Table I. The constants of the measuring instruments are also given, so that one may compute the corresponding results for himself. The results obtained are given in Tables II and III and charted 
TABLE II

RESULTS

\begin{tabular}{|c|c|c|}
\hline Frequency & $\begin{array}{c}\text { 7-inch sphere gaps; } \\
\text { inches }\end{array}$ & $\begin{array}{l}\text { Kilovolts by in- } \\
\text { struments }\end{array}$ \\
\hline 123,000 & 0.427 & 20.2 \\
\hline " & 0.429 & 19.8 \\
\hline “ & 0.452 & 20.0 \\
\hline “ & 0.540 & 24.7 \\
\hline$"$ & . 0.673 & 33.0 \\
\hline “ & 0.693 & 33.0 \\
\hline$山$ & 0.695 & 32.7 \\
\hline$u$ & 0.704 & 33.0 \\
\hline 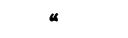 & 0.738 & 32.7 \\
\hline “ & 0.784 & 35.7 \\
\hline$\approx$ & 0.800 & 35.8 \\
\hline$a$ & 0.811 & 36.6 \\
\hline “ & 0.820 & 37.0 \\
\hline “ & 0.821 & 37.0 \\
\hline « & 0.863 & 40). 7 \\
\hline " & 0.878 & 40.7 \\
\hline “ & 1.03 & 47.5 \\
\hline “ & 1.04 & 44.7 \\
\hline$"$ & 1.06 & 48.8 \\
\hline “ & 1.06 & 48.0 \\
\hline “ & 1.09 & 49.8 \\
\hline " & 1.09 & 49.4 \\
\hline$"$ & 1.10 & 48.5 \\
\hline$a$ & 1.10 & 50.4 \\
\hline$"$ & 1.16 & 51.8 \\
\hline
\end{tabular}

TABLE III

RESULTS

\begin{tabular}{|c|c|c|}
\hline Frequency & $\begin{array}{c}\text { 7-inch sphere gaps; } \\
\text { inches }\end{array}$ & $\begin{array}{l}\text { Kilovolts, by in- } \\
\text { struments }\end{array}$ \\
\hline 255,000 & 0.261 & 13500 \\
\hline “ & 0.253 & 13300 \\
\hline “ & 0.248 & 13250 \\
\hline “ & 0.371 & 19060 \\
\hline “ & 0.378 & 18460 \\
\hline “ & 0.382 & 18400 \\
\hline “ & 0.337 & 17670 \\
\hline “ & 0.404 & 20770 \\
\hline “ & 0.898 & 43300 \\
\hline$\alpha$ & 0.817 & 42600 \\
\hline “ & 0.886 & 42200 \\
\hline " & 0.920 & 42300 \\
\hline “ & 0.901 & 41900 \\
\hline “ & 0.931 & 43700 \\
\hline " & 0.934 & 44200 \\
\hline 612,500 & 0.320 & $1+950$ \\
\hline “ & 0.263 & 12300 \\
\hline “ & 0.285 & 13200 \\
\hline “ & 0.242 & 12480 \\
\hline “ & 0.250 & 12200 \\
\hline
\end{tabular}


in Fig. 5. It is noted that the 123,000-cycle values, when the fluctuating character of the source is ignored, locate a right line relation for the sphere gaps and discharge voltages; and that such line when extended passes through the origin. See curve I, Fig. 5. All values determined at 123,000, 255,000 and 612,500 cycles locate curve II, Fig. 5, when errors due to the fluctuation of the source are largely climinated.

Curve III, Fig. 5, was drawn for comparison; it was located

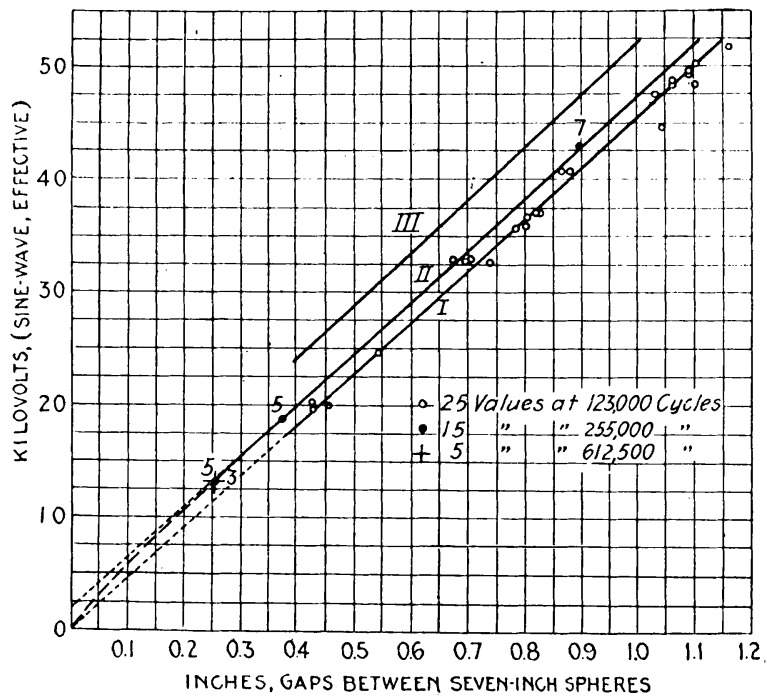

Fig. 5

I. Seven-inch sphere-gap discharge voltage at 123,000 cycles, fluctuations of source ignored. 25 determinations.

II. Ditto. 25 determinations at 123,000 cycles, 15 determinations at 255,000 cycles and 5 determinations at 612,500 cycles. Fluctuations of source eliminated by giving maximum weight to minimum gaps for corresponding voltages.

III. 25-, 37.5- and 50-centimeter sphere-gap discharge voltages at 25 cycles determined by Chubb and Fortescue, Proc. A. I. E. E., Feb. 1913, pp. 635-6.

with the obscrvations made by Chubb and Fortescue for 25 cycles discharge voltages and $25,37.5-$ and $50-\mathrm{cm}$. sphere gaps. These observations are recorded on pp. 635-6 of the Fcbruary, 1913, Proceedings A. I. E. E. The high-frequency voltages are almost uniformly 4.5 kilovolts below the 25 -cycle voltages. No cause for this difference could be found in our methods of measurements. We belicve that both curves have been correctly located. 


\section{Conclusions}

1. The seven-in. $(17.7-\mathrm{cm}$.) sphere-gap) discharge voltages at frequencies ranging from 123,000 to 255,000 cycles per second are given by the expression

$$
e=45.5 l+2
$$

for the gap range of 0.4 to $1.1 \mathrm{in}$. ( 1 to $25.6 \mathrm{~mm}$.), whercin the value of $e$ is in kilovolts, r.m.s., and the value of the sphere gap, $l$, is in inches at ordinary atmospheric conditions: Temp. $18 \mathrm{deg}$. cent.; Barom. 30 in.

2. Correspondingly at 612,500 cycles per second, 13.06 kilovolts, r.m.s., discharge through a seven-in. sphere-gap length of 0.252 inch.

3. Necdle gaps are not practicable for the determination of high-frequency high voltages. The points are promptly melted and burned. Only gaps between blunt points can be employed. For these, No. 14 B. \& S. gage, square-cnded copper wires were used. The measured voltages were found to be about one-half of the corresponding voltages at 60 cycles.

\section{ACKNOWLEDGEMENTS}

The writers could not have undertaken the present work without special equipment, provided as follows:

Mr. Cyril F. Elwell presented the Department of Electrical Enginecring, Leland Stanford Junior University, with a fivekilowatt Poulsen arc oscillating-current gencrator. Later on the Federal Telegraph Company generously exchanged this generator for a powerful $12-\mathrm{kw}$. Poulsen-Federal arc-gencrator, together with a variable condenser and special minor supplies such as litzen draht, carbons and bushings. Invaluable advice and suggestions were rendered in the earlier stages of the work by Mr. Elwell and Mr. Leonard F. Fuller. 